\title{
FITTING ISOCHRONE OF HYADES STAR CLUSTER BY BUILDING COLOUR MAGNITUDE DIAGRAM (CMD)
}

\author{
Annisa Novia Indra Putri ${ }^{1}$ \\ ${ }^{1}$ Institut Teknologi Sumatera, Lampung Selatan, Lampung
}

\begin{abstract}
:
Sky survey of Star cluster from many projects have many informations about our Galaxy, Milky Way. Hyades star cluster is the nearest open cluster and one of the best-studied star cluster. Its location about 153 light-years away from the Solar System consist of roughly spherical group of hundreds of stars. Earlier information from this cluster can be used to constraint other paramaters such as structuer, composition, dynamic formation, and evolution of Galaxy. Aim this research is fitting model of simple Colour Magnitude Diagram (CMD) with isochrone from Hyades data using monte caro method including binary star fraction effect and fotometry uncertainty. Basic parameter Hyades data is taken from WEBDA while isochrone Hyades data is taken from Padova site. The result from this research is limiting magnitude instrument observing Hyades around 16 mag. There are some points of Hyades data forming new main sequence under real main sequence which fit with model due to closer distance of Hyades star cluster from the Sun.
\end{abstract}

Keywords: Open cluster, Hyades, Color Magnitude Diagram, Isochrone

\section{Introduction}

Stars are formed from big cloud called nebula and as group called cluster. Star cluster is assumed as Simple Stellar Population (SSP). SSP is an object formed at the same time which has same chemical composition, distance, and reddening. Theoretically, SSP is called isochrone. Isochrone from a cluster has a same age but different mass. Star mass correlate with magnitude of star, bigger mass of star so the star will be brighter. Hyades star cluster is one of kind open cluster in the Universe and the nearest open cluster from the Sun. Hyades is located at Taurus constellation consisting of a roughly spherical group of hundred of stars sharing the same age, distance, chemical characteristics, and motion through the space. In this research purpose to do modelling simple Colour Magnitude Diagram (CMD) from open cluster of Hyades then fitting to isochrone Hyades data using monte carlo method.

\subsection{Data}

\section{Data and Research Method}

Building Color Magnitude Diagram (CMD) need some initial parameters such as isochrone, Initial Mass Function (IMF), reddening, age interval, and metalicity. Initial basic parameter Hyades is taken from WEBDA [1] shown at Table 1. While isochrone of Hyades data use isochrone information at Padova site [2] based on some publications [3,4,5].

\begin{tabular}{|l|l|}
\hline \multicolumn{1}{|c|}{ Parameter } & \multicolumn{1}{c|}{ Value } \\
\hline RA (J2000) & $04^{\mathrm{h}} 26^{\mathrm{m}} 54^{\mathrm{s}}$ \\
\hline Declination (J2000) & $+15^{\circ} 52^{\prime} 00^{\prime \prime}$ \\
\hline Galaxy Longitude & $180 \mathrm{\circ}^{\circ} 064$ \\
\hline Galaxy Latitude & $-22, \mathrm{o}^{\circ} 43$ \\
\hline Distance (pc) & 45 \\
\hline Reddening (mag) & 0,01 \\
\hline Distance Modulus (mag) & 3,30 \\
\hline
\end{tabular}


\begin{tabular}{|l|l|}
\hline Age $[\mathrm{Log}]$ & 8,896 \\
\hline Metalicity & $+0,17$ \\
\hline
\end{tabular}

\subsection{Method}

Reddening occurs as a result from Interstellar Matter (ISM) between the object and observer. Beside reddening, cluster distance also affects apparent magnitude value. Reddening value can change the position of star point at Color Magnitude Diagram (CMD) to the horizontal directoin while the distance changes the position of the point at $\mathrm{CMD}$ in vertical direction. Reddening of the photometric system can be expressed in color excess, $\mathrm{E}(\mathrm{B}-\mathrm{V})$, which the amount of absorption at visual wavelength is expressed by this equation :

$$
A_{v}=R_{v} E(B-V)
$$

with $A_{v}$ is absorption value and $R_{v}$ is reddening value at visual wavelength. $R_{v}$ value is approximately 2.5 for the general diffuse ISM [6].

Star distribution with the mass formed simultaneously is defined as the Initial Massa Function (IMF). This function describes dynamic evolution, chemistry, brightness of star cluster, and the initial process of forming cluster. The simplest model from IMF is written in the form power law function :

$$
\frac{d N}{d m}=X=k m^{-\alpha}
$$

with $\mathrm{k}$ is normalization factor and $\alpha$ is power law index which use Salpeter index value, 2.35 [7]. By interpreting IMF as probability density function, it is possible to choose a star mass at a certain interval mass using :

$$
\begin{aligned}
& X(m)=\int k m^{-\alpha} d m \\
& k=\frac{1-\alpha}{m_{u}^{1-\alpha}-m_{l}^{1-\alpha}}
\end{aligned}
$$

Mass function depend on random value $\mathrm{X}$ can be written as :

$$
m(X)=\left[m_{l}^{1-\alpha}+\left(\frac{1-\alpha}{k}\right) X\right]^{\frac{1}{1-\alpha}}
$$

$X$ is a random value, $m_{l}$ and $m_{u}$ is low and upper interval star mass from Hyades star cluster. At CMD, observing data usually shows different widening interval colour from theoretical model. Widening colour is caused by photometric error and groupping binary star effect visually. In this research add photometric error value 0.03 [8].

Fitting isochrone by building CMD model can be done with these steps [9]: (1) Getting Isochrone Hyades data, (2) Making star randomly using monte carlo simulation method, (3) Adding group of binary star effect, (4) Inputing distance and reddening parameter, and (5) Adding photometric error effect.

\section{Result and Analysis}

The monte carlo simulation results of Colour Magnitude Diagram (CMD) model in the star-making process by including photometric uncertainties show at Figure 1. Black and green colour indicate CMD model without and including binary star fraction effect while brown-cross points are Hyades star cluster data from observation. In Figure 1 shows Hyades data only spreadaround 16 mag which indicate limiting magnitude of instrument for observing Hyades 16 mag. Beside that,There are some Hyades data points forming 'new main sequence line' under main sequence which fit with model. Appearance 'new main sequence line' due to distance of Hyades star cluster which is relatively close to the observer so the distance from each member of Hyades cluster can be determined more easily and accurately than 
the further clusters where the distance from further clusters can be assumed as overall distance of cluster members.

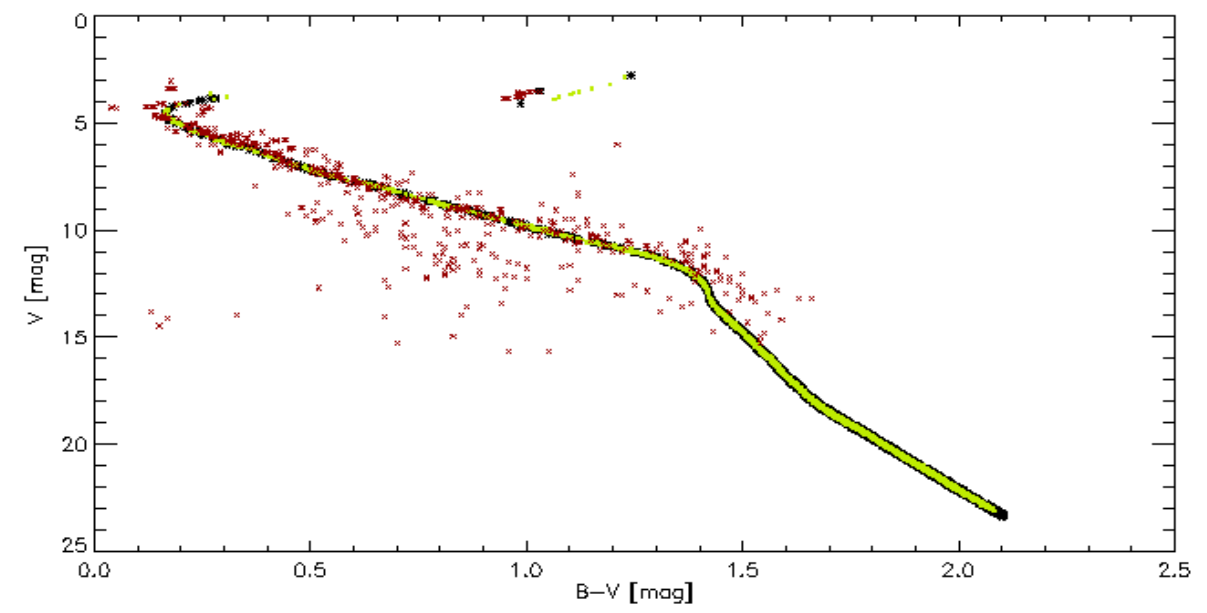

Figure 1 Fitting CMD model with Hyades star cluster data. Black and green colour are CMD model without and including binary star fraction effect while brown-cross points are Hyades star cluster data from observation.

From Figure 1, model CMD and Hyades cluster data are fitted with Hyades isochrone by adding distance and reddening parameter showed at Figure 2 including photometric error 0.03 where red line is Hyades isochrone. Bigger photometric uncertainties can cause bigger spread and dispersion of CMD (showed at Figure 3).

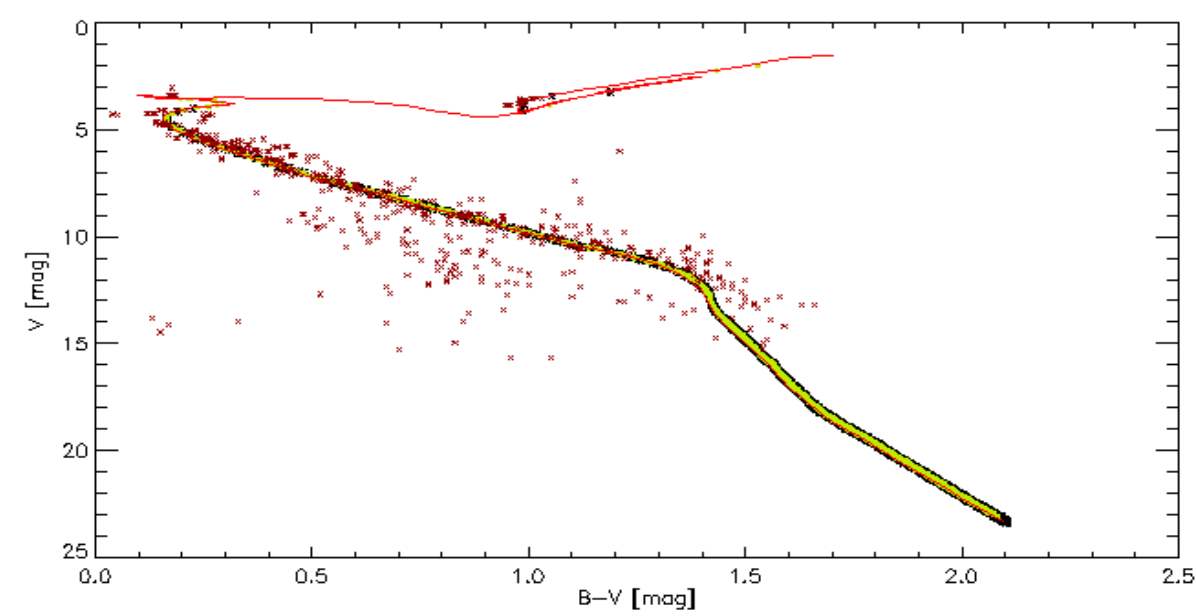

Figure 2 Fitting Hyades isochrone with CMD model and Hyades star cluster data including photometric error 0.03. Red line is Hyades cluster isochrone. 


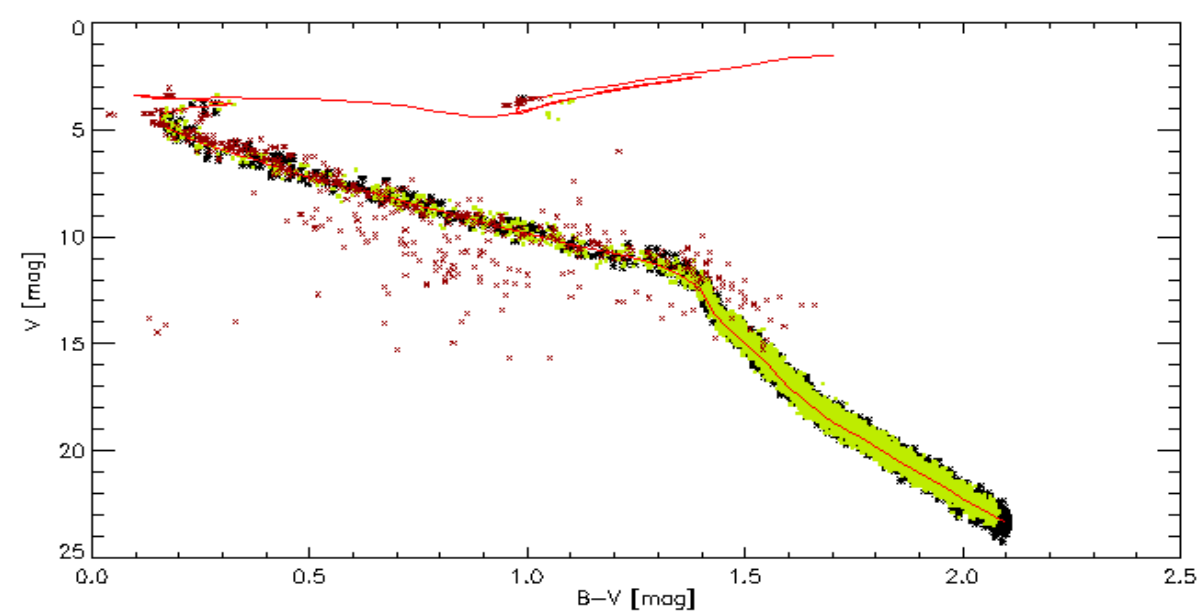

Figure 3 Fitting Hyades isochrone with CMD model and Hyades star cluster data including photometric error 0.3. Red line is Hyades cluster isochrone.

\section{Conclusion}

Hyades star cluster is the nearest open cluster from the Sun and one of subject for the best studiedcluster. Earlier information about Hyades can be used to constraint any parameter of Universe. In this research, Hyades star cluster is fitted with isochrone by building Color Magnitude Diagram (CMD). Hyades star cluster shows new main sequence line under main sequence which fit with model. Occurances of this line because of the distance of Hyades star cluster which is relatively close to the observer. CMD model with Hyades data also are fitted with Hyades isochrone by adding photometric error value 0.03 and 0.3 . Fitting with bigger photometric error shows bigger dispersion of CMD model.

\section{References}

[1] WEBDA page for open cluster Hyades, September 2013, http://www.univie.ac.at/webda/cgibin/ocl_page.cgi?cluster=hyades.

[2] CMD 3.0 input form, October 2012, http://stev.oapd.inaf.it/cgi-bin/cmd.

[3] B. Aringer, L. Girardi, W. Nowotny, P. Marigo, and A. Bressan, "Synthetic photometry for M and K giants and stellar evolution: hydrostatic dust-free model atmospheres and chemical abundances", Monthly Notices of The Royal Astornomical Society, vol. 457, pp. 3611-3628, January 2016.

[4] A. Bressen, P. Marigo, L. Girardi, B. Salasnich, C. Dal Cero, S. Rubele, A. Nanni, "PARSEC: stellar tracks and isochrones with PAdova and Triesete stellar evolution code", Monthly Notices of The Royal Astornomical Society, vol. 427, pp. 127-145, August 2012.

[5] C. Yang, G. Leo, B. Alessandro, M. Paola, B. Mauro, and K. Xong, "Improving PARSEC models for very low mass stars", Monthly Notices of The Royal Astornomical Society, vol. 444, pp. 2525-2543, September 2014.

[6] D. M. Nataf et al, "Reddening and extinction toward the galactic bulge from OGLE III: the inner milky way's Rv $\sim 2.5$ exctinction curve", The Astrophysical Journal Letters, pp. 769-788, June 2013.

[7] M. S. Oey, "On the origin of the salpeter slope for the initial mass function", The Astrophysical Journal Letters, pp.739:746, October 2011.

[8] S. Cassisi and M. Salaris, "Old stellar populations", Germany: Wiley, 2013.

[9] D. T. Wahyuningtyas, "Metode fitting isochrone untuk penentuan parameter umur dan jarak gugus terbuka kasus: blanco 1, NGC 1245, dan Ru 27", Tesis S2 Astronomi Institut Teknologi Bandung, Bandung, Indonesia, 2010. 